\title{
Erratum to: Redefining phenotypes associated with mitochondrial DNA single deletion
}

\author{
Michelangelo Mancuso ${ }^{1} \cdot$ Daniele Orsucci ${ }^{1} \cdot$ Corrado Angelini $^{2} \cdot$ Enrico Bertini $^{3}$ • \\ Valerio Carelli ${ }^{4}$ - Giacomo Pietro Comi ${ }^{5}$ Maria Alice Donati ${ }^{6}$ - Antonio Federico ${ }^{7}$. \\ Carlo Minetti $^{8}$ - Maurizio Moggio ${ }^{9}$ Tiziana Mongini ${ }^{10} \cdot$ Filippo Maria Santorelli $^{11}$. \\ Serenella Servidei $^{12} \cdot$ Paola Tonin $^{13} \cdot$ Antonio Toscano $^{14} \cdot$ Claudio Bruno $^{8}$. \\ Luca Bello $^{2}$ - Elena Caldarazzo Ienco ${ }^{1}$ - Elena Cardaioli $^{7} \cdot$ Michela Catteruccia $^{3}$. \\ Paola Da Pozzo ${ }^{7}$ - Massimiliano Filosto ${ }^{17}$. Costanza Lamperti ${ }^{16}$ - Isabella Moroni ${ }^{15}$. \\ Olimpia Musumeci $^{14}$ - Elena Pegoraro ${ }^{2}$ - Dario Ronchi ${ }^{5}$ - Donato Sauchelli ${ }^{12}$. \\ Mauro Scarpelli ${ }^{13} \cdot$ Monica Sciacco $^{9} \cdot$ Maria Lucia Valentino $^{4} \cdot$ Liliana Vercelli $^{10}$. \\ Massimo Zeviani $^{16} \cdot$ Gabriele Siciliano $^{1}$
}

Published online: 14 November 2015

(C) Springer-Verlag Berlin Heidelberg 2015

\section{Erratum to: J Neurol (2015) 262:1301-1309 DOI 10.1007/s00415-015-7710-y}

The correct affiliations of Prof. Valerio Carelli and Dr. Maria Lucia Valentino are:

Department of Biomedical and Neuromotor Sciences, University of Bologna and IRCCS Istituto delle Scienze Neurologiche di Bologna, Bologna, Italy.

The online version of the original article can be found under doi:10.1007/s00415-015-7710-y.

Michelangelo Mancuso

mancusomichelangelo@gmail.com

1 Neurological Clinic, University of Pisa, Via Roma 67, 56126 Pisa, Italy

2 Neurological Clinic, University of Padova, Padua, Italy

3 Unit for Neuromuscular and Neurodegenerative Disorders, Laboratory of Molecular Medicine,, Bambino Gesù Children's Hospital, IRCCS, Rome, Italy

4 Department of Biomedical and Neuromotor Sciences, University of Bologna and IRCCS Istituto delle Scienze Neurologiche di Bologna, Bologna, Italy

5 Neuroscience Section, Neurology Unit, Department of Pathophysiology and Transplantation (DEPT), Dino Ferrari Centre, IRCCS Foundation Ca' Granda Ospedale Maggiore Policlinico, University of Milan, Milan, Italy

6 Meyer Children's Hospital, University of Florence, Florence, Italy

7 Department of Medicine, Surgery and Neurosciences, University of Siena, Siena, Italy
8 Neuropediatric and Center of Myology and Neurodegnerative Disorders, Istituto Giannina Gaslini, Genoa, Italy

9 Neuromuscular Unit, Dino Ferrari Centre, Fondazione IRCCS Ca' Granda Ospedale Maggiore Policlinico, Milano, University of Milan, Milan, Italy

10 Department of Neuroscience, University of Turin, Turin, Italy

11 IRCCS Stella Maris, Pisa, Italy

12 Institute of Neurology, Catholic University, Rome, Italy

13 Neurological Clinic, University of Verona, Verona, Italy

14 Department of Neurosciences, University of Messina, Messina, Italy

15 Child Neurology Unit, The Foundation "Carlo Besta" Institute of Neurology-IRCCS, Milan, Italy

16 Unit of Molecular Neurogenetics, The Foundation "Carlo Besta" Institute of Neurology-IRCCS, Milan, Italy

17 Neurological Clinic, University Hospital Spedali Civili, Brescia, Italy 